\title{
Flow Evaluation and Simulation Fuel in the Injector of a MEP Engine When Using Fuels Alternatives
}

\section{Evaluación y Simulación del Flujo de Com- bustible en el Inyector de un Motor MEP Al Emplear Combustibles Alternativos}

I International Congress of Science and Technology Morona Santiago-CICTMS 2020

Corresponding Author:

Diego Javier Punina Guerrero dpunina.mdm@uisek.edu.ec

Published: 29 August 2021

Production and Hosting by Knowledge E

(c) Diego Javier Punina Guerrero et al. This article is distributed under the terms of the Creative Commons Attribution License, which permits unrestricted use and redistribution provided that the original author and source are credited.
S OPEN ACCESS

\section{Diego Javier Punina Guerrero', Carlos Alberto Gallardo Naula ${ }^{2}$, and Josencka Dariana Sarmiento Anchundia ${ }^{3}$}

${ }^{1}$ Universidad Internacional SEK, Facultad de Arquitectura e Ingeniería, Quito, Ecuador

${ }^{2}$ Universidad Internacional SEK, Facultad de Arquitectura e Ingeniería, Quito, Ecuador

${ }^{3}$ Universidad Técnica Estatal de Quevedo UTEQ, Facultad de Ciencias de la Ingeniería, Quevedo, Ecuador

\section{Abstract}

This work showed the use of alternative fuels as a means of generating energy using biodiesel based on frying oil through an evaluation and simulation of the fuel flow in the injectors of a MEP engine. With the application of the experimental method, tests were carried out on normal diesel, biodiesel with mixtures B10, B20 and B100 in the mechanical injectors to observe their opening pressure, closing time, spray angle and their dripping. With the data obtained from the tests in the laboratory, the statistical method was used to verify if they were in the usual operating range of the injectors to proceed with the simulation in ANSYS software, evaluating the behavior of the fluid inside the injector, in addition to obtain values of pressures and velocities in the flow of the fluid within it. In this way, alternatives were evaluated and proposed in the use of biodiesel as an alternative fuel, choosing the geometry of 5 outlets because the pressure remained constant with the use of diesel fuel and the frying oil biofuel, with all the combinations, which which indicated that a variation was necessary and not other complementary accessories on the engine.

Keywords: Injectors, MEP engine, biofuel, diesel

\section{Resumen}

Este trabajo mostró el uso de combustibles alternativos como medio de generación de energía usando biodiesel a base de aceite de frituras mediante una evaluación y simulación del flujo de combustible en los inyectores de un motor MEP. Con la aplicación del método experimental se realizó pruebas en el diésel normal, biodiesel con mezclas B10, B20 y B100 en los inyectores mecánicos para observar su presión de apertura, tiempo de cierre, ángulo de pulverización y el goteo de los mismos. Con los datos obtenidos de las pruebas en el laboratorio, se empleó el método estadístico para verificar si se encontraban en el rango habitual de funcionamiento de los inyectores para proceder a la simulación en software ANSYS, evaluando el comportamiento del fluido dentro del inyector, además de obtener valores de presiones y velocidades en el flujo del fluido dentro del mismo. De tal forma, se evaluó y propuso alternativas en el uso del biodiesel como combustible alternativo, escogiendo la geometría de 5 salidas debido que la presión permaneció constante con el uso del combustible diésel y el biocombustible de aceite de frituras, con todas las combinaciones, lo cual indicó que era necesario una variación y no otros accesorios complementarios en el motor. 


\section{Introducción}

Gran parte de los países se realiza investigaciones para encontrar alternativas al diésel como combustible. Alguna de las cuales se encuentran orientadas al desarrollo de aceites vegetales como fuente de abaratamiento y con mayor densidad de energía que los aceites vegetales convertidos, causando menor impacto ambiental [1].

La importancia de la velocidad de inyección del diésel ha motivado el desarrollo de este trabajo, donde, después de evaluar diferentes métodos y equipos comerciales para determinar la tasa de inyección, se decidió utilizar el método comúnmente conocido como método Bosch, debido a sus ventajas y fácil operación, en él se presenta la integración y configuración de un sistema, sus procedimientos y resultados para determinar la tasa de inyección al modificar diferentes parámetros de inyección con diferentes combustibles alternativos [2].

El combustible diésel debe permitir la marcha suave del motor con poco golpeteo. El combustible debe permanecer líquido a bajas temperaturas, de tal modo que el motor arrancará fácilmente y marchará suavemente por lo cual el diésel sirve como lubricante para la bomba de inyección e inyectores, por lo tanto, deberá tener adecuadas propiedades de lubricación [3].

El biodiesel posee un beneficio principal el cual es su bajo contenido de azufre, esto se debe a que en su composición química existe oxígeno, así la combustión es más completa, disminuyendo la emisión de partículas, monóxido de carbono no quemado, y otros contaminantes.

El aceite de fritura usado es una de las alternativas con mejores perspectivas en la producción de biodiesel, ya que es la materia prima más barata, y con su utilización se evitan los costes de tratamiento como residuo. Ecuador es un gran consumidor de aceites vegetales, centrándose el consumo en el aceite de palma [4].

La evaluación del comportamiento del flujo dentro del inyector mediante simulación en el software ANSYS permite garantizar el buen funcionamiento del mismo al usar biocombustible, para lo cual se realiza pruebas de funcionamiento y presión en los inyectores para determinar los valores de funcionalidad. A su vez, evaluar el funcionamiento de los inyectores mediante el uso de biodiesel alternativo al 10\% (B10), $20 \%$ (B20) y 100\% (B100) con la geometría propuesta del ducto de fluido [5].

Finalmente, la simulación del biodiesel en el inyector en el software ANSYS nos permite observar las variaciones del fluido del inyector y llegar a las conclusiones correspondientes [6]. 


\section{Materiales y Métodos}

\subsection{Método experimental}

Para que los inyectores desempeñaran correctamente su función, se debió probar y regular en un equipo probador de inyectores, el cual fue el H17.8 diésel.

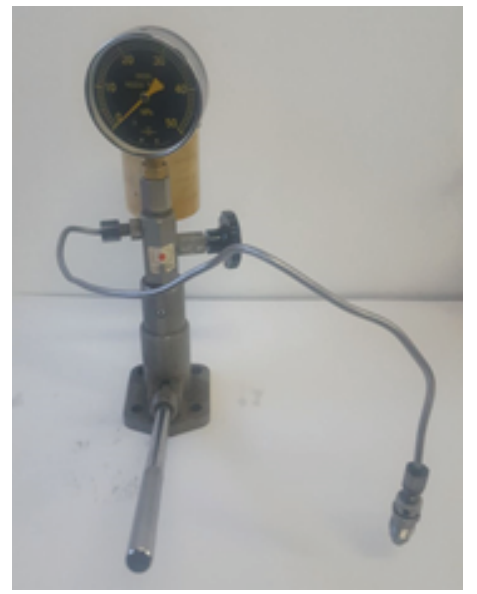

\section{Figure 1}

Comprobador de inyectores modelo H17.8. (Fuente: Autor.)

Para la fase experimental, la toma de datos se realizó mediante pruebas en el inyector mecánico con la finalidad de obtener los valores para realizar la simulación. Para cada una de las pruebas, se realizó con el combustible diésel y los biocombustibles de aceite de frituras B10, B20 y B100, las cuales fueron:

1. Presión de apertura del inyector;

2. Prueba de estanqueidad;

3. Cálculo del ángulo de chorro de inyección.

En la prueba de presión de apertura del inyector, se mantuvo abierto el medidor de presión donde se aplicó lentamente la palanca del probador. La presión de apertura alcanzada del inyector fue aquella que marca en el manómetro, siendo la presión en la que se abrió el inyector y pasó el fluido.

La prueba de estanqueidad se realizó con el medidor de presión abierto, aplicando lentamente la palanca. Se observó el manómetro y se ajustó la presión a un nivel de 20 bar inferior a la presión de apertura anterior. Con esa presión, no goteó ni una gota de aceite de calibración durante 10 segundos.

El cálculo del ángulo de pulverización se lo realizó con los datos que se obtienen del inyector. A éste se le colocó a una determinada altura donde el chorro realizó una marca en un papel blanco, determinando la longitud del diámetro y en función de estos datos se procedió a calcular el ángulo de pulverización, realizando varias veces la prueba hasta obtener un promedio. 


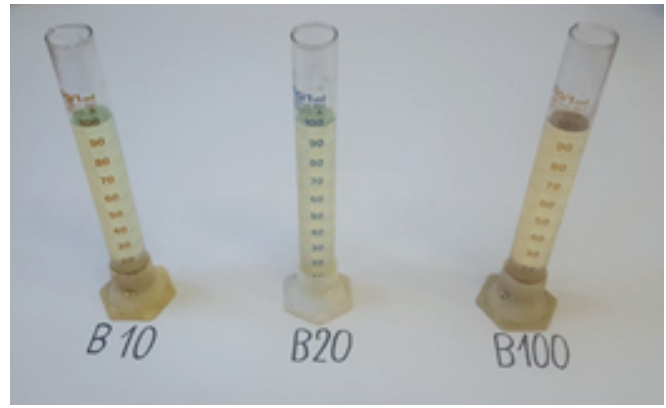

\section{Figure 2}

Biocombustible mezclas B10, B20 y B100. (Fuente: Autor.)

\subsection{Método estadístico}

Para conocer la fiabilidad de la información, se procedió a realizar un análisis de datos. Por lo que mediante una gráfica de control para la media se pudo establecer una estimación de $\mu$. En base a este valor se alcanzó un límite superior de control y un límite inferior, los cuales se consiguieron con las siguientes ecuaciones:

$$
\begin{aligned}
& L S C_{\bar{X}}=\bar{X}+A_{2} \bar{R} \\
& L I C_{\bar{X}}=\bar{X}-A_{2} \bar{R},
\end{aligned}
$$

donde:

$$
\bar{X}=\frac{\sum \bar{X}}{k} .
$$

Por otra parte, para los cálculos de la variación en el rango de las muestras fueron calculados con las gráficas de control para la dispersión, siendo las fórmulas:

$$
\begin{aligned}
& L S C_{R}=D_{4} \bar{R} \\
& L I C_{R}=D_{3} \bar{R},
\end{aligned}
$$

donde $\bar{R}$ fue el rango promedio de los rangos muéstrales y A2 fue una constante basada en el tamaño de la muestra. Los valores de A2 se encontraron en la tabla de factores críticos de las gráficas o cartas de control. $Y \bar{X}$ fue la media de los valores.

Con los datos obtenidos en las pruebas experimentales en los inyectores, se llevó a cabo el diseño en el software Solidworks y su análisis en ANSYS con la finalidad de variar la geometría de manera que se pudo observar el comportamiento de la velocidad, turbulencia y presiones de los fluidos tanto para la geometría de 3 salidas, 5 salidas y de la variación de los diámetros a la salida del flujo en el inyector.

\section{Resultados}

Para las presiones de apertura del diésel, B10, B20 y B100, las pruebas realizadas con el diésel en comparación con el biocombustible de aceite de frituras B10, B20 y B100 
se obtuvieron los resultados que se muestran en la Tabla 1, también se muestran los valores promedios y rango para realizar el cálculo de límite superior e inferior, tanto de rangos como de medias.

\section{Table 1}

Presiones de apertura del inyector.

\begin{tabular}{|c|c|c|c|c|c|c|}
\hline Prueba & Diésel (MPa) & $\mathrm{B} 10$ (MPa) & B20 (MPa) & B100 (MPa) & Promedio (X) & Rango (R) \\
\hline 1 & 16 & 21 & 22 & 25 & 21 & 9 \\
\hline 2 & 16 & 20 & 23 & 25 & 21 & 9 \\
\hline 3 & 16 & 21 & 23 & 24 & 21 & 8 \\
\hline 4 & 16 & 21 & 22 & 24 & 20.75 & 8 \\
\hline 5 & 15 & 20 & 22 & 25 & 20.5 & 10 \\
\hline 6 & 16 & 20 & 23 & 25 & 21 & 9 \\
\hline 7 & 17 & 21 & 22 & 25 & 21.25 & 8 \\
\hline 8 & 16 & 20 & 23 & 25 & 21 & 9 \\
\hline 9 & 17 & 21 & 22 & 24 & 21 & 7 \\
\hline 10 & 15 & 20 & 23 & 24 & 20.5 & 9 \\
\hline Prom & 16 & 20.5 & 22.5 & 24.6 & $x=20.9$ & $R=8.6$ \\
\hline
\end{tabular}

Donde B10 es biodiésel alternativo al 10\%, B20 es biodiésel alternativo al 20\% y B100 es biodiesel alternativo al $100 \%$.

Fuente: Autor.

Con la aplicación de las ecuaciones (1) y (2) se observó que no existe valor que salga de los límites de control, por lo cual los valores obtenidos en la fase experimental fueron correctos.

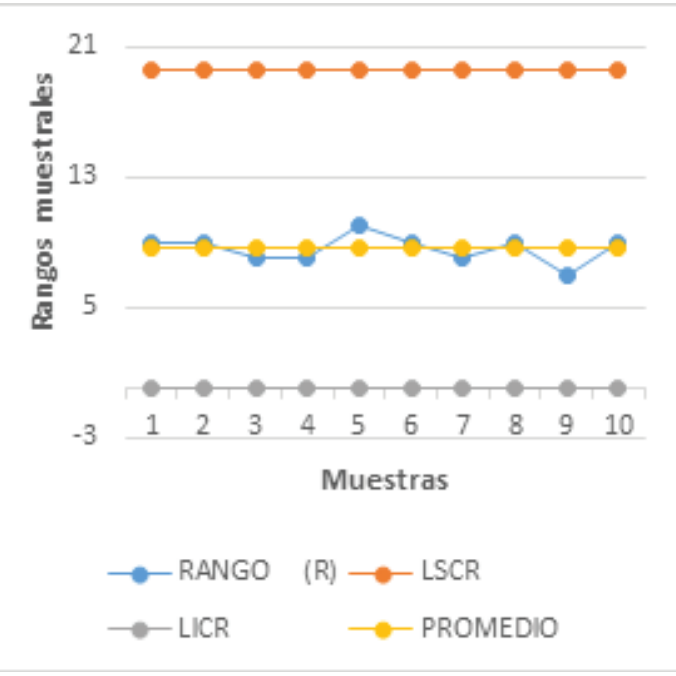

Figure 3

Gráfica de control de rango de carga absoluta. (Fuente: Autor.) 
Para el cálculo del ángulo de pulverización, se colocó el inyector en el probador de inyectores diésel a una altura de $15 \mathrm{~cm}$, siendo un valor considerado debido a que el ángulo de pulverización efectivo es de $60 \%$ de su totalidad.

Con las consideraciones descritas anteriormente, las pruebas en el laboratorio del Instituto Tecnológico Superior Guayaquil al inyector mecánico, se obtuvieron las siguientes variaciones del ángulo de pulverización cuando trabaja con diésel y biocompatible combustible a las diferentes mezclas, cuyos datos promedio para cada biodiésel se obtuvieron realizando diez pruebas respectivamente.

\section{Table 2}

Resultados del ángulo de pulverización para el diésel, B10, B20 y B100.

Combustible
Diésel
B10
B20
B100

Ángulo de pulverización $\left(^{\circ}\right.$ )
16.80
19.59
20.63
22.29

Fuente: Autor.

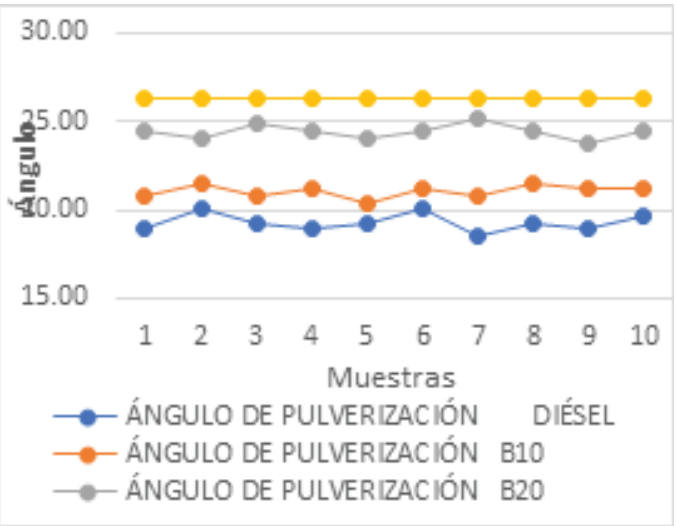

\section{Figure 4}

Resultados del ángulo de pulverización para el diésel, B10, B20 y B100. (Fuente: Autor.)

Los datos obtenidos demostraron una tendencia polinómica de la distribución de datos donde la variante fue la relación de mezcla del biocombustible.

Por otra parte, las condiciones de borde para el análisis de la velocidad, presión e intensidad de turbulencia del fluido se consideró las siguientes condiciones de borde obtenidas en el método experimental, las cuales se introdujeron en el software ANSYS. Estos análisis se realizaron para el inyector de 3 salidas, de 5 salidas y para la disminución en el diámetro. 


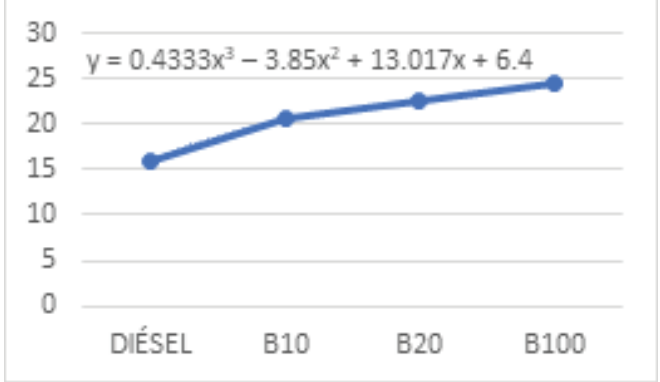

Figure 5

Tendencia polinómica de las mezclas de biocombustible. (Fuente: Autor.)

Table 3

Valores de simulación con geometría 3 salidas.

\begin{tabular}{|c|c|c|c|c|c|c|c|c|}
\hline Fluido & $\begin{array}{l}\text { Densidad } \\
\left(\mathrm{kg} / \mathrm{m}^{3}\right)\end{array}$ & $\begin{array}{l}\text { Viscosidad } \\
\text { cinemática } \\
\text { (cst) }\end{array}$ & $\begin{array}{l}\text { Viscosidad } \\
\text { dinámica }(\mathrm{cp})\end{array}$ & $\begin{array}{l}\text { Presión de } \\
\text { salida } \\
\text { (MPa) }\end{array}$ & $\begin{array}{l}\text { Velocidad } \\
\text { de entrada } \\
(\mathrm{m} / \mathrm{s})\end{array}$ & $\begin{array}{l}\text { Velocidad } \\
\text { máxima } \\
(\mathrm{m} / \mathrm{s})\end{array}$ & $\begin{array}{l}\text { Presión } \\
\text { máxima } \\
\text { (MPa) }\end{array}$ & $\begin{array}{l}\text { Intensidad } \\
\text { de } \\
\text { turbulencia }\end{array}$ \\
\hline Diesel & 730 & 5 & 0.0024 & 16 & 0.3 & 8.20 & 16.04 & 1.13 \\
\hline $\mathrm{B} 10$ & 468 & 3.44 & 0.00160992 & 20.5 & 0.3 & 8.20 & 20.53 & 1.14 \\
\hline $\mathrm{B} 20$ & 480 & 3.45 & 0.001656 & 22.5 & 0.3 & 8.20 & 22.52 & 1.14 \\
\hline B100 & 882 & 6.069 & 0.00535285 & 24.6 & 0.3 & 8.19 & 24.65 & 1.28 \\
\hline
\end{tabular}

Fuente: Autor

Table 4

Valores de simulación con geometría 5 salidas.

\begin{tabular}{l|l|} 
Fluido & $\begin{array}{l}\text { Densidad } \\
\left(\mathbf{k g} / \mathbf{m}^{3} \mathbf{)}\right.\end{array}$ \\
\hline Diesel & 730 \\
\hline B10 & 468 \\
\hline B20 & 480 \\
\hline B100 & 882 \\
\hline
\end{tabular}

$\begin{aligned} & \text { Viscosidad } \\ & \text { cinemática } \\ & \text { (cst) }\end{aligned}$
5
3.44
3.45
6.069

$\begin{aligned} & \text { Viscosidad } \\ & \text { dinámica (cp) }\end{aligned}$
0.0024
0.00160992
0.001656
0.00535285

\begin{tabular}{|l|l|}
$\begin{array}{l}\text { Presión de } \\
\text { salida } \\
\text { (MPa) }\end{array}$ & $\begin{array}{l}\text { Velocidad } \\
\text { de entrada } \\
\text { (m/s) }\end{array}$ \\
\hline 16 & 0.3 \\
\hline 20.5 & 0.3 \\
22.5 & 0.3 \\
24.6 & 0.3 \\
\hline
\end{tabular}

$\begin{aligned} & \text { Velocidad } \\ & \text { máxima } \\ & (\mathbf{m} / \mathbf{s})\end{aligned}$
5.07
5.10
5.13
5.18

\begin{tabular}{|l|l|}
$\begin{array}{l}\text { Presión } \\
\text { máxima } \\
\text { (MPa) }\end{array}$ & $\begin{array}{l}\text { Intensidad } \\
\text { de } \\
\text { turbulencia }\end{array}$ \\
\hline 16.018 & 0.91 \\
\hline 16.020 & 0.94 \\
\hline 16.021 & 0.98 \\
\hline 16.024 & 1.07 \\
\hline
\end{tabular}

Fuente: Autor.

\section{Table 5}

Valores de simulación con disminución en el diámetro de salida del inyector.

\begin{tabular}{|l|l|l|}
\hline Fluido & $\begin{array}{l}\text { Densidad } \\
\left(\mathbf{k g} / \mathbf{m}^{3}\right)\end{array}$ & $\begin{array}{l}\text { Viscosidad } \\
\text { cinemática }\end{array}$ \\
\hline (cst)
\end{tabular}

$\begin{aligned} & \text { Viscosidad } \\ & \text { dinámica (cp) }\end{aligned}$
0.0024
0.00160992
0.001656
0.00535285

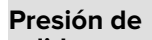
salida (MPa)

16

20.5

22.5

24.6

Fuente: Autor.

\begin{tabular}{|l|l|}
$\begin{array}{l}\text { Velocidad } \\
\text { de entrada } \\
\text { (m/s) }\end{array}$ & $\begin{array}{l}\text { Velocidad } \\
\text { máxima } \\
\text { (m/s) }\end{array}$ \\
\hline 0.3 & 11.62 \\
\hline 0.3 & 11.65 \\
\hline 0.3 & 11.67 \\
\hline 0.3 & 11.89 \\
\hline
\end{tabular}

Presión máxima

(MPa)

16.08

20.52

20.53

24.70

$\begin{aligned} & \text { Intensidad } \\ & \text { de } \\ & \text { turbulencia }\end{aligned}$
1.53
1.55
1.56
1.79




\section{Discusión}

Realizado los análisis del diésel y biodiesel en los inyectores de 3 salidas y 5 salidas, y las variaciones de los diámetros de la salida de flujo se pudo observar que las variaciones de presiones en la apertura del inyector al usar diferentes combustibles, se concluyó que se encuentran bajo control y no existen variaciones que salgan fuera de los límites de control, pero el biocombustible requiere mayor presión de apertura debido a su mayor densidad.

Además, lo que analíticamente representa una tendencia polinómica de la distribución de datos donde la variante es la relación de mezcla del biodiesel, en comparación con (Guerrero, 2018) al usar biocombustible de origen vegetal presenta la misma tendencia de forma polinómica. El ángulo de pulverización aumentó al momento de trabajar con el biodiesel de mayor densidad, siendo un parámetro que podría afectar el rendimiento del motor y sus emisiones, dato que coincide con lo mencionado según Guerrero (2018) que el ángulo varia debido a la densidad del combustible, el mismo que es un parámetro muy importante según Pavía (2015), ya que el chorro influye directamente en la combustión de la mezcla aire combustible Por otra parte, las velocidades del fluido en la geometría de 3 salidas (Tabla 3) la variación más significativa es entre el biocombustible B20 y el diésel, existiendo una variación del 0.12\%, además que la máxima velocidad existe en la punta del inyector debido al área de comparación al resto de su geometría, por lo que tiende a aumentar. Al aumentar el número de salidas a 5 , la máxima velocidad se dio con el biocombustible a $5.18 \mathrm{~m} / \mathrm{s}$ mientras que la mínima fue para el fluido diésel con $5.07 \mathrm{~m} / \mathrm{s}$. Comparando con la geometría original, se produjo un decremento del $36.8 \%$ en velocidad, datos que en comparación según Rojas (2012) la velocidad de inyección durante un ciclo termodinámico es esencial debido a que es una información importante como parámetro de control de la formación de la mezcla aire-combustible.

La variación del diámetro de salida de $0.7 \mathrm{~mm}$ a $0.5 \mathrm{~mm}$ de la geometría original de 3 salidas, se presentó una mayor velocidad de $11.89 \mathrm{~m} / \mathrm{s}$ en el biocombustible B100, pero su turbulencia fue de 1.79. En la geometría de 5 salidas, la mayor velocidad fue de $5.18 \mathrm{~m} / \mathrm{s}$ la cual se encuentra en el biocombustible B100, la misma que presenta mayor presión y mayor turbulencia en la variación de sus diámetros con una turbulencia de 1.07 .

Con los datos obtenidos de las geometrías, se observó que la mejor propuesta es la variación de diámetros en la geometría original de 3 salidas datos que coinciden con lo mencionado según Pavía (2015) que menciona que cuando la velocidad es la máxima la presión será la mínima como se puede observar en los resultados obtenidos Tabla 6.

\section{Conclusiones}

La evaluación y simulación de combustible en el inyector mecánico de un motor MEP al emplear combustibles alternos como el biodiesel de aceite de frituras en mezclas B10, B20 y B100, se mostró que variando la geometría del inyector en la salida se 
Table 6

Valores máximos de velocidades presiones e intensidad de turbulencia de los combustibles.

\begin{tabular}{l|l}
\hline Geometría & Velocidad m/s \\
\hline Geometría original 3 salidas & 8.20 con B10 \\
\hline Geometría 5 salidas & 5.18 con B100 \\
\hline Geometría variación de diámetros & 11.89 con B100
\end{tabular}

Fuente: Autor.

Presión Mpa
24.65 con B100
16.024 con B100
24.7 con B100

Turbulencia

1.26 con $\mathrm{B} 100$

1.07 con $\mathrm{B} 100$

1.79 con $\mathrm{B} 100$

obtiene beneficios en la inyección del combustible. A su vez, la simulación del flujo del combustible en el inyector mecánico arrojó que su velocidad máxima será d 11.89 $\mathrm{m} / \mathrm{s}$ al usar el biocombustible B100 a una presión máxima de $24.7 \mathrm{MPa}$ con un flujo turbulento de 1.79; lo cual generaría una detonación previa en el cilindro y causaría daños mecánicos en el motor y, por otra parte, también los valores de turbulencia no son beneficiosos debido a que el diésel y el biocombustible tienden a calentarse. Acorde a los resultados obtenidos en la fase experimental y simulación, el uso del biocombustible B10 sería el más adecuado porque presenta condiciones de trabajo óptimas.

Se propusieron dos geometrías diferentes, la de 3 y 5 salidas, obteniéndose que presenta mejores resultados la variación a 5 conductos de salida debido a que la presión se mantuvo constante en $16 \mathrm{MPa}$, además que el uso del diésel y el biocombustible no se vio alterado a pesar que presenta una menor velocidad que puede ser compensada en el cambio del ángulo de adelanto de inyección.

\section{Agradecimientos}

Agradezco infinitamente a mis padres y hermanos por siempre haber confiado en mí, por brindarme siempre sus consejos y el apoyo brindado, quienes me motivaron siempre a seguir adelante y luchar por mis sueños, a la Universidad Internacional SEK por los conocimientos brindados en la obtención del título de post grado y a los docentes quienes han sabido transmitir toda su información y experiencias de una manera extraordinaria con dedicación y responsabilidad, los mismos que dejan grandes recuerdos.

\section{References}

[1] Delalibera HC, Campolina N, Neto PHW, Ralisch R. Performance of a diesel engine fueled with a preheated blend of soybean oil and petrodiesel. SciELO Brasil. 2012; 32(6):1058-1067.

[2] Armas O, Mata C, Martínez-Martínez S. Effect of diesel injection parameters on instantaneous fuel delivery using a solenoid-operated injector with different fuels. Revista Facultad de Ingeniería Universidad de Antioquia. 2012;62:64.

[3] Castro-Martínez C, Beltrán-Arredondo LI, Ortiz-Ojeda JC. Producción de Biodiesel y bioetanol: ¿Una alternativa sustentable a la crisis energética? Ra Ximhai. 2012;8(3):10.

[4] Vargas FAA. Producción y caracterización de biodiesel de palma y de aceite reclicado mediante un proceso batch y un proceso continuo con un reactor helicoidal Tarragona. Universitat Rovira I Virgili; 2010. 
[5] Guerra FJR, Tamayo OJY. Estudio de la mezcla óptima diesel aceite de palma usada como combustible de un motor a diesel. Sangolqui: Escuela Politécnica del Ejercito; 2007.

[6] Lazo OR, Rojas LR. Diseño asistido por computador. Industrial Data. 2006;9(1):7-1.

[7] Arredondo LEL, VFJR. Obtención de Biodiesel a partir de diferentes tipos de grasa residual de origen animal. Luna Azul. 2013;(36).

[8] Berbel RL. Estudio de la viscosidad y densidad de diferentes aceites para su uso como biocombustible. Catalunya: Universitat Politecnica de Catalunya; 2010.

[9] Boretti A. Use of bio-ethanol and bio-diesel the key solution for a more sustainable road transport. SAE Technical Paper; 2011 September.

[10] Cedeño EAL, Rocha-Hoyos J, Alvear PS, Barboza JM. Producción e Impacto del Biodiesel. INNOVA Research Journal. 2017;2(7):59-76.

[11] Dorado MP, Pinzi S, Haro A, Font R, Garcia-Olmo J. Visible and NIR Spectroscopy to assess biodiesel quality: Determination of alcohol and glycerol traces. Elsevier. 2011 March;90(6);2321-2325.

[12] Marcelo LP, Alejandro FS, C RRA. CFD como una herramienta de diseño para productos. XV Congreso Internacional Anual de la SOMIM; 2009; Ciudad de México, Mexico.

[13] Parra P. Cálculo de un sistema de inyección multifase para motores diesel. Valencia: Universidad Politécnica de Valencia; 2014.

[14] Vrublevskyi O, Lasynovych B. Methods of diagnosing vehicle diesel injectors. Automobile Transport. 2016;38:55-61. 